Revue

Revue de l'histoire des religions

de Ihistoire des religions

1 | 2016

Varia

Maureen C. MILLER, Clothing the Clergy. Virtue and

Power in Medieval Europe, c. 800-1200

Ithaca-Londres, Cornell University Press, 2014

Patrick Henriet

\title{
CpenEdition
}

Journals

Édition électronique

URL : http://journals.openedition.org/rhr/8510

DOI : 10.4000/rhr.8510

ISSN : 2105-2573

Éditeur

Armand Colin

Édition imprimée

Date de publication : 1 mars 2016

Pagination : 118-122

ISBN : 978-2-200-93059-2

ISSN : 0035-1423

Référence électronique

Patrick Henriet, « Maureen C. MILlen, Clothing the Clergy. Virtue and Power in Medieval Europe, c. 800-1200

», Revue de l'histoire des religions [En ligne], 1 | 2016, mis en ligne le 04 avril 2016, consulté le 25

septembre 2020. URL : http://journals.openedition.org/rhr/8510; DOI : https://doi.org/10.4000/rhr. 8510

Ce document a été généré automatiquement le 25 septembre 2020.

Tous droits réservés 


\section{Maureen C. MILLER, Clothing the Clergy. Virtue and Power in Medieval Europe, c. 800-1200}

Ithaca-Londres, Cornell University Press, 2014

Patrick Henriet

\section{RÉFÉRENCE}

Maureen C. MILLER, Clothing the Clergy. Virtue and Power in Medieval Europe, c. 800-1200, Ithaca-Londres, Cornell University Press, 2014, 25,5 cm, 286 p., 89,95 \$, ISBN 978-0-8014-4982-6.

1 Les objets n'ont pas dans les sociétés traditionnelles la même fonction que dans nos sociétés post-industrielles et capitalistes. C'est assurément un truisme de le rappeler : encore faut-il, lorsqu'on les étudie dans un environnement particulier, comprendre leur valeur symbolique, leurs usages et les enjeux qui accompagnent ces derniers. Le livre de Maureen C. Miller s'y emploie par l'étude des vêtements ecclésiastiques entre l'époque carolingienne et la fin approximative d'une longue période "grégorienne » (le concile de Latran IV étant cité à plusieurs reprises, il eût été plus juste de faire figurer 1215 dans le titre). Ce sont les vêtements du clergé séculier qui sont ici pris en compte.

2 L'ouvrage tout entier est placé sous le signe d'un hommage à l'« immensely learned Jesuit » Joseph Braun ( $† 1947)$, célèbre pour ses travaux sur l'autel mais aussi auteur d'une monumentale étude sur le vêtement liturgique (Die liturgische Gewandung im Occident und Orient, 1907). Cependant, si Maureen Miller sait mettre à profit, tout en l'actualisant, l'immense documentation rassemblée par Braun, elle donne à son étude une tout autre orientation : il s'agit de comprendre comment la construction de l'Église médiévale et celle de son apparence sont liées. C'est un passionnant projet, mené de façon synthétique et nerveuse, qui emporte généralement l'adhésion. 
$3 \mathrm{Au}$ cœur de l'ouvrage, une constatation qui porte essentiellement sur les habits liturgiques: alors que dans l'Antiquité et le haut Moyen Âge les vêtements des clercs restent relativement sobres, ils deviennent considérablement plus ornés à partir de l'époque carolingienne. L'origine de ce tournant capital serait à chercher dans le monde anglo-saxon ou dans la Francia du nord-est, là où apparaissent pour la première fois des vêtements de soie brodés d'or. Les résistances à cette évolution, présentes sous Charlemagne et sous Louis le Pieux, tomberaient définitivement vers le milieu du $\mathrm{Ix}^{\mathrm{e}}$ siècle, comme en témoignerait la représentation des clercs dans certains manuscrits : ainsi la célèbre page de dédicace de la «Bible à Vivien ", réalisée en 845 pour Charles le Chauve, ou encore le sacramentaire (aujourd'hui conservé à Autun) de Raganaldus, datable des mêmes années. Cette évolution semble incontestable dans les grandes lignes. Peut-être, cependant, est-elle déjà entérinée avant les années 840. En effet, les textes d'Alcuin ou de l'Astronome utilisés p. 115 pour suggérer une hostilité initiale du pouvoir carolingien à l'enrichissement des habits liturgiques ne me semblent pas probants : ils concernent en réalité non pas le culte, mais la tenue quotidienne des clercs, par exemple lorsqu'ils viennent au palais. Quoi qu'il en soit, les vêtements somptueusement ornés se répandent ensuite partout en Europe, sans doute à des rythmes quelque peu différents. Les sources sont évidemment très lacunaires et les chercheurs sont à la merci des hasards de la conservation : enluminures, mosaïques, fresques et bien entendu, mais plus rarement, vêtements conservés dans un musée. Une image bien connue peut résumer à elle seule le mouvement qui vient d'être décrit : c'est celle de l'évêque Ecclesius offrant son église à la Vierge sur une mosaïque de Saint-Vital de Ravenne. L'œuvre est du milieu du $\mathrm{VI}^{\mathrm{e}}$ siècle mais elle a été restaurée au XII ${ }^{\mathrm{e}}$ siècle. On a alors rajouté à l'habit épiscopal des franges richement ornées et une dalmatique magnifiquement décorée.

4 Toute cette évolution est longuement étudiée par Miller dans le contexte de la réforme dite grégorienne. La splendeur des vêtements liturgiques participe alors d'un processus de différenciation qui permet d'affirmer les hiérarchies et en même temps de marquer la différence de nature qui sépare les clercs des laïques. Tout le problème, ici, est évidemment de savoir ce qu'il faut entendre par "réforme ", car le monde ottonien, dans lequel l'empereur dirige clairement son Église (que l'on accepte ou non l'expression et le concept de «Reichskirchensystem»), a atteint un degré de raffinement peut-être sans précédent dans l'ornementation des habits cultuels. Rien n'indique par ailleurs que les clercs fidèles à l'empereur durant la querelle dite « des investitures » aient utilisé des vêtements différents de ceux de leurs adversaires. Miller voit bien cette difficulté et parle très justement d'un "shared language of power » (i.e. partagé entre tous les clercs, quel que soit le degré de proximité avec le centre réformateur romain). Cette notion passionnante aurait d'ailleurs pu être développée. Elle implique en effet que tous les clercs, au-delà de leur engagement dans un camp ou dans un autre, participaient d'une même évolution de fond qui affectait les structures de l'Église et ses rapports au monde.

5 Le passage à des habits travaillés, ornés voire exubérants, s'est également accompagné d'une réflexion qui, le plus souvent, prenait place dans les traités liturgiques. On trouve donc des interprétations allégoriques chez Bède (déjà), chez Raban Maur, chez Amalaire de Metz et chez Walafrid Strabon, puis aux XI ${ }^{\mathrm{e}}$ et $\mathrm{XII}^{\mathrm{e}}$ siècles chez Bruno de Segni, chez Jean d'Ivry ou encore chez Sicard de Crémone, sans oublier bien sûr l'œuvre de Durand de Mende, dont on sait l'immense succès au xiII ${ }^{e}$ siècle. Une absence 
surprend toutefois dans les développements de l'auteur, précisément sur un point qui lui aurait permis d'aller encore un peu plus loin: on ne trouve en effet dans le livre aucune réflexion sur le nom de certains de ces traités. Or dans plusieurs cas, les titres montrent à quel point le vêtement était désormais considéré comme central dans le dispositif liturgique. Sicard de Crémone (né vers 1155 et non vers 1185, p. 58) intitule son ouvrage Mitrale, ce qui se passe de commentaire. Plus saisissant est assurément le nom de ce best-seller que fut le Rationale divinorum officiorum de Durand de Mende († 1296). Rationale de ratio, dans le contexte du développement des écoles urbaines et de la scolastique? Certainement pas. Miller consacre de nombreuses et belles pages au rationale d'Exode 28 (traduction de Jérôme), un pectoral qui fut porté pour la première fois par Aaron et après lui, dans l'Ancien Testament, par le grand prêtre. Ce rationale avait sa place sur l'ephod (ou amict). Il était tissé, entre autres, de fils d'or, et il était garni de pierres précieuses qui portaient les noms des douze tribus d'Israël. Or, sans aucun doute possible, le traité de Guillaume Durand tire son nom de cette pièce de tissu qui a très tôt suscité des commentaires allégoriques (en particulier dans la Regula pastoralis de Grégoire le Grand) : l'auteur explique en effet longuement dans le prologue que son livre porte ce titre en référence au « rational du jugement que le pontife de la loi portait sur sa poitrine ».

6 À côté des traités liturgiques, certains vêtements conservés et certaines enluminures témoignent de constructions allégoriques extrêmement complexes et réellement fascinantes. Citons en particulier cette extraordinaire image du Codex dit "d'Uta » (Regensburg, ca.1025, aujourd'hui Munich, Clm 13601), qui montre saint Erhard en train de dire la messe revêtu du rational. Sur celui-ci, on peut clairement lire les mots IHPAPXHIA (Hiérarchie), Veritas et Doctrina (lesquels renvoient à Exode 28). Du côté des vêtements conservés, comment ne pas mentionner cet autre rationale, du milieu du $\mathrm{XI}^{\mathrm{e}}$ siècle, aujourd'hui conservé à Bamberg ? Le commentaire de Miller est ici pleinement convaincant. Peut-être, et c'est Bamberg qui me pousse à émettre cette proposition, eût-il cependant été fécond d'introduire dans la discussion le manteau de couronnement d'Henri II ( $† 1024)$, conservé dans la même ville: bien des points évoqués à propos des habits cléricaux pourraient certainement s'appliquer aussi aux manteaux d'apparat et de couronnement des empereurs ottoniens et saliens, dont le modèle était lui aussi vétérotestamentaire (Sagesse 18, 24). Ce qui amènerait sans doute à poser un peu différemment, pour cette époque, la question des relations entre clercs et « laïques ». Le manteau d'Henri II fut d'ailleurs ultérieurement retouché en vue d'une utilisation liturgique.

7 On ne peut jamais convaincre en tout : il est donc un point d'une certaine importance, développé par Miller, sur lequel je souhaite brièvement m'arrêter. Au cours du XII siècle, en effet, les clercs auraient été tellement intéressés par leurs vêtements qu'ils en seraient venus à inventer des habits d'un type nouveau, condamnés ensuite par la hiérarchie ecclésiastique. Une affaire intra-ecclésiale, donc. L'exemple abouti de ce processus serait la cappa manicata, décrite par Miller comme un vêtement spécifiquement clérical destiné aux sorties extérieures et dont le port fut condamné à Latran IV (canon 16). Cette interprétation s'appuie sur la conviction (étayée par une recherche dans la base de données des Monumenta Germaniae Historica) que la cappa serait toujours un habit clérical. Or les exemples donnés par l'auteur (Vie de Thomas Becket par William Fitzstephen en particulier) montrent bien que cette cappa manicata (une grande cape que l'on enfilait par le cou, qui était pourvue d'ouvertures latérales 
permettant de sortir les bras et sur laquelle on avait cousu, vers l'arrière et en guise d'ornements, les manches qui donnaient son nom à l'habit) était perçue comme d'essence laïque. Par ailleurs les usages du terme cappa pour désigner un habit profane porté par des laïques sont en réalité très fréquents (il suffit de se reporter à Du Cange, s. v. cappa): dans les Coutumes de Chartreuse consignées par Guigues $\mathrm{I}^{\mathrm{er}}$ dans les années 1120, au moment de sa profession, le novice retire la cappa pour revêtir la cuculle (24.1). L'idée que l'Église condamnait des pratiques vestimentaires spécifiquement cléricales mises au point par des clercs me semble donc au moins en partie fausse. L'interdiction de la cappa manicata doit être comprise comme une injonction de plus (il y en avait eu beaucoup depuis des siècles) visant à empêcher les clercs de s'habiller et de se comporter comme d'arrogants laïques. Toujours dans ce long passage sur la cappa manicata, signalons une lecture erronée d'une enluminure du Décret de Gratien (reproduite p. 47) : ce ne sont pas deux clercs qui discutent, comme il est dit p. 46, mais un clerc et un laïque, sans doute le magistrat civil dont il est question dans le texte de Gratien (causa XI).

Ce livre est une mine d'informations, il est richement et élégamment illustré, il formule des propositions stimulantes et souvent convaincantes. D'autres peuvent être discutées, nous l'avons vu. Je n'en ai donné ici qu'un aperçu général, passant sous silence certains développements qui me semblent un peu moins centrés sur le vrai sujet (ainsi le rôle des femmes dans la fabrication des vêtements). Le "vrai sujet ", me semble-t-il, c'est la question de l'inscription progressive et toujours plus accentuée de l'Église dans la matière et dans le monde. Le processus qui a amené les évêques et les prêtres à porter des vêtements extraordinairement luxueux lors des célébrations liturgiques est le même, par exemple, que celui par lequel l'institution ecclésiale a valorisé les bâtiments et les propriétés qui permettaient à ceux-ci d'exister. Ce livre peut donc être considéré, même s'il ne le dit pas vraiment, comme un jalon de plus dans l'histoire d'une Église incorporée, ancrée dans des réalités terrestres qui, si elles étaient présentées comme les signes de réalités invisibles, n'en étaient pas moins nécessaires et donc valorisées. Il reste une question que je n'aurai pas l'audace de prétendre traiter ici: quelle est réellement, dans cette construction, le rôle de la réforme dite grégorienne? Révolution et moment de rupture, ou bien point d'aboutissement, voire simple étape? Dans l'histoire du vêtement liturgique, le tournant capital est amorcé au moins deux siècles avant Grégoire VII. Cela ne va pas dans le sens d'une « révolution grégorienne ». Mais ce ne sont là que timides suggestions, qu'il conviendrait de reprendre et d'étayer. Dans l'immédiat, lisons ce beau livre.

\section{AUTEURS}

\section{PATRICK HENRIET}

École Pratique des Hautes Études, Paris. 